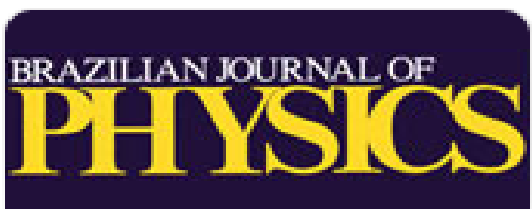

Brazilian Journal of Physics

ISSN: 0103-9733

luizno.bjp@gmail.com

Sociedade Brasileira de Física

Brasil

Avila Aoki, Manuel

Optimal Estimate of a Pure Qubit State from Uhlmann-Josza Fidelity

Brazilian Journal of Physics, vol. 42, núm. 1-2, 2012, pp. 1-5

Sociedade Brasileira de Física

Sâo Paulo, Brasil

Available in: http://www.redalyc.org/articulo.oa?id=46423428001

How to cite

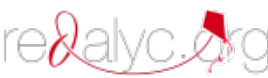

Complete issue

- More information about this article

Journal's homepage in redalyc.org

Scientific Information System Network of Scientific Journals from Latin America, the Caribbean, Spain and Portugal Non-profit academic project, developed under the open access initiative 


\title{
Optimal Estimate of a Pure Qubit State from Uhlmann-Josza Fidelity
}

\author{
Manuel Avila Aoki
}

Received: 3 August 2011 / Published online: 21 January 2012

(c) Sociedade Brasileira de Física 2012

\begin{abstract}
In the framework of collective measurements, efforts have been made to reconstruct onequbit states. Such schemes find an obstacle in the no-cloning theorem, which prevents full reconstruction of a quantum state. Quantum Mechanics thus restricts us to obtaining estimates of the reconstruction of a pure qubit. We discuss the optimal estimate on the basis of the Uhlmann-Josza fidelity, respecting the limitations imposed by the no-cloning theorem. We derive a realistic optimal expression for the average fidelity. Our formalism also introduces an optimization parameter $L$. Values close to zero imply full reconstruction of the qubit (i. e., the classical limit), while larger L's represent good quantum optimization of the qubit estimate. The parameter $L$ is interpreted as the degree of quantumness of the average fidelity associated with the reconstruction.
\end{abstract}

Keywords Quantum • State estimate • Tomography • Uhlmann-Josza fidelity $\cdot$ Optimization

\section{Introduction}

Quantum mechanical measurements being probabilistic, even the assessment of measurable quantities calls for estimation methods. In Quantum Information, state estimation is important from both the theoretical and the practical points of view. In many cases it results

M. A. Aoki $(\varangle)$

Centro Universitario UAEM Valle de Chalco, UAEMex,

Valle de Chalco, CP 56615, Edo. de México, México

e-mail:manvlk@yahoo.com important to know the initial state in order to implement the specific quantum processing of an algorithm in a quantum computer $[1,2]$. The estimation relies on physical reconstruction of the qubit state, a task to which much work has been devoted in recent years [3]. The common approach is to perform measurements on an ensemble of identically prepared systems and use such measurements for a probabilistic reconstruction of the qubit state. The greater the probability, the better the reconstruction (estimation). With an infinite ensemble of copies, the state would be exactly determined. In practice, however, we have access to a limited number of copies and the state can be only approximately determined [4]. In order to achieve the best reconstructed state, the optimal experimental strategy, readily feasible in the laboratory, is to make individual Von Neumann measurements $[5,6]$. For this protocol scarce analytical result are known. General, simultaneous measurements on all copies of the qubit would therefore be desirable. Unfortunately, such collective measurements are very difficult to implement.

In their attempt to reconstruct a qubit state these approaches find a serious obstacle in the no-cloning theorem $[7,8]$, which forbids exact reconstruction of a quantum state [9]. Here we estimate a pure qubit [10] in an optimal way without contravening the no-cloning theorem.

\section{Uhlmann-Josza Fidelity}

Our approach is based on tomographic-inspired schemes [11, 12]. Consequently, we are assuming that we are given an ensemble of $N$ copies of the qubit state, 
which we denote by $|\vec{n}\rangle$. Here $\vec{n}$ is the unique vector on the Bloch sphere satisfying the equality

$\sigma_{n}=|\vec{n}\rangle\langle\vec{n}|=(1+\vec{n} \cdot \vec{\sigma}) / 2$,

where $\vec{\sigma}$ are the usual Pauli matrices. Let us denote by $x$ the set of outcomes of a set of measurements on the $N$ copies of the qubit [13] and by $|\vec{M}(x)\rangle$ the estimate for $|\vec{n}\rangle$.

Central to our analysis is the fidelity, which quantifies the quality of the estimation. Reference [3] defined the fidelity as the overlap $|\langle\vec{n} \mid \vec{M}(x)\rangle|^{2}=$ $[1+\vec{n} \cdot \vec{M}(x)] / 2$. In Quantum Information, however, this quantity offers less confidence than the UhlmannJosza fidelity [14-16]. For this reason, we shall be using the latter quantifier, whose mathematical expression is

$F=[\operatorname{Tr}(\sqrt{\sqrt{\rho} \sigma \sqrt{\rho}})]^{2}$,

where $\rho$ and $\sigma$ are two different density matrices. The Uhlmann-Josza fidelity $F$ is a measure between quantum states. For a pure state $|\psi\rangle$ and an arbitrary state $\rho$, it is the overlap between $|\psi\rangle$ and $\rho$, that is, $F=[\operatorname{Tr}(\sqrt{\langle\psi|\rho| \psi\rangle})]^{2}[16]$.

Given any two states on the Bloch sphere $|\vec{M}(x)\rangle$ and $|\vec{n}\rangle$, if they are related through an arbitrary rotation gate

$|\vec{M}(x)\rangle=\mathcal{R}\left(\theta_{x}\right)|\vec{n}\rangle$,

where

$\mathcal{R}\left(\theta_{x}\right)=\exp \left(-i \theta_{x} \vec{m} \cdot \vec{\sigma} / 2\right)|\vec{n}\rangle$,

with $\vec{m}$ the unit vector along the corresponding rotation vector, so that (3a) can be written in the form

$|\vec{M}(x)\rangle=\exp \left(-i \theta_{x} \sigma_{z}\right)|\vec{n}\rangle$

or more explicitly,

$|\vec{M}(x)\rangle=\left(\begin{array}{ll}e^{-i \theta_{x} / 2} & 0 \\ 0 & e^{i \theta_{x} / 2}\end{array}\right)|\vec{n}\rangle$,

then the no-cloning theorem is contradicted since the state can be reconstructed completely, i.e., $F=1$.

This can be seen as follows. From (3), the density matrix associated with $|\vec{M}(x)\rangle$ will be

$\rho_{M}=|\vec{M}(x)\rangle\langle\vec{M}(x)|$,

or

$\rho_{M}=\left(\begin{array}{ll}e^{-i \theta_{x} / 2} & 0 \\ 0 & e^{i \theta_{x} / 2}\end{array}\right) \sigma_{n}\left(\begin{array}{ll}e^{i \theta_{x} / 2} & 0 \\ 0 & e^{-i \theta_{x} / 2}\end{array}\right)$, with $\sigma_{n}$ as defined by (1). Since the rotational invariance of the scalar product allows us to choose $\vec{n}$ along the $z$-direction, $\sigma_{n}$ and $\rho_{M}$ have the same eigenvalues, $\lambda_{1}=(1+|\vec{n}|) / 2=1$ and $\lambda_{2}=(1-|\vec{n}|) / 2=0$. Substitution of $\lambda_{1}$ and $\lambda_{2}$ in (2) yields $F=1$.

In a special case the overlap fidelity of [3] is reproduced. Indeed, if the estimate is such that

$|\vec{M}(x)\rangle\langle\vec{M}(x)|=\frac{1+M_{3} \sigma_{3}}{2}$,

then the Uhlmann-Josza fidelity is

$F_{3}=\frac{1+\vec{n} \cdot \vec{M}}{2}$.

To see this, notice that the eigenvalues of (6) are $\left(1 \pm M_{3}\right) / 2=\left(1 \pm \cos \theta_{x}\right) / 2$. The rotational invariance of the scalar product implies that $\vec{n} \cdot \vec{M}=M_{3}=\cos \theta_{x}$, and (6) follows.

In general, however, even a unitary vector $\vec{n}$ is insufficient to make the Uhlmann-Josza fidelity equal to the overlap fidelity in [3], as the following argument shows. If the estimate is such that

$|\vec{M}(x)\rangle\langle\vec{M}(x)|=\frac{1+M_{1} \sigma_{1}+M_{2} \sigma_{2}}{2}$

then the Uhlmann-Josza fidelity is

$F_{12}=\frac{1+\sin \theta_{x}}{2}$,

where $\sin \theta_{x}=|\vec{n} \times \vec{M}(x)|$.

To prove (8), we note that the eigenvalues of (1) are 1 and 0 while the eigenvalues of (7) are $\left(1 \pm \sqrt{M_{1}^{2}+M_{2}^{2}}\right) / 2=\left(1 \pm \sin \theta_{x}\right) / 2$, where $M_{1}=$ $\sin \theta_{x} \cos \varphi_{x}$ and $M_{2}=\sin \theta_{x} \sin \varphi_{x}$. Substitution of these quantities in (2) then completes the proof.

The fidelity $F_{12}$ in (8) has azimuthal symmetry and hence a single degree of freedom. For realistic estimates, which do not exhibit this symmetry, $F_{12}$ is not the most general fidelity. In general, the only allowed constraint is $|\vec{M}(x)|=1$, and the problem has two degrees of freedom. For this reason, the fidelity $F_{123}$ in [17] is redundant and was not used for the estimate of a qubit. In the more general case, the estimate is such that

$|\vec{M}(x)\rangle\langle\vec{M}(x)|=\frac{1+M_{1} \sigma_{1}+M_{3} \sigma_{3}}{2}$.

The fidelity then becomes

$F_{13}=\frac{1}{2}\left(1+\sqrt{M_{1}^{2}+M_{3}^{2}}\right)=\frac{1}{2}\left(1+\sqrt{1-M_{2}^{2}}\right)$ 
or

$$
F_{13}=\frac{1}{2}\left(1+\sqrt{1-\sin ^{2} \theta_{x} \sin ^{2} \varphi_{x}}\right) .
$$

Equation (10) follows from (6), since the eigenvalues of (1) are 1 and 0 , while the eigenvalues of (9) are $\lambda_{1,2}=$ $\frac{1}{2}\left(1 \pm \sqrt{M_{1}^{2}+M_{3}^{2}}\right)$.

It is instructive to discuss the implications of this result. We recall that the fidelity is a real parameter satisfying $0 \leq F \leq 1[18,19]$. From Eq. (10), since $0 \leq$ $M_{2} \leq 1$, it then follows that the more general fidelity lies in the interval

$\frac{1}{2} \leq F_{13} \leq 1$.

From (10) we also see that $M_{2}=0$ implies $F_{13}\left(M_{2}=\right.$ $0)=1$, in contradiction with the no-cloning theorem, since the qubit state would then be fully reconstructed. We will deal with this difficulty in Section 4.

\section{Average Fidelity}

Our goal then becomes to optimize the average fidelity over the initial probability and all possible outcomes

$\langle F\rangle=\sum_{x=000 \cdots 00}^{2^{N}} F_{13}\left(\theta_{x}, \varphi_{x}\right) p_{n}(x)$,

where $F_{13}\left(\theta_{x}, \varphi_{x}\right)$ is given by (10).

Within the approaches of [20-23] the expression for $\langle F\rangle$ differs from (12). The difference arises because the authors do not use the Uhlmann-Josza fidelity. Instead, they search to reconstruct the qubit state on the basis of a measurement scheme. This task is nontrivial, due to the no-cloning theorem. Furthermore, to maximize $\langle F\rangle$ it would be necessary to make $N=\infty$ measurements, which is impossible in practice. This understood, we try to optimize (12), in lieu of maximizing the fidelity. This amounts to making $1 \ll N<\infty$ measurements.

On the other hand, we observe that quantum probability distribution function must satisfy $p_{n}\left(M_{2}=\right.$ $0)=p_{n}\left(F_{13}=1\right)=0$, while a classical one should satisfy $p_{n}\left(M_{2}=0\right)=p_{n}\left(F_{13}=1\right)=1$ (the latter equality would contradict the no-cloning theorem since $F_{13}=1$ implies a fully reconstructed quantum state). Furthermore, for a large enough number of measurements a gaussian distribution peaked around $M_{2}=0$ subtracted from unity becomes a reasonable assumption. Thus, account taken of these two arguments, we consider a consistent quantum probability function given by the following expression
Definition 1 The probability function associated with the average fidelity in (12) is

$$
\begin{aligned}
p_{n}\left(\theta_{x}, \varphi_{x}\right) & =A(x)\left(1-e^{-M_{2}^{2}}\right) \\
& =A(x)\left(1-e^{-\sin ^{2} \theta_{x} \cos ^{2} \varphi_{x}}\right)
\end{aligned}
$$

where $A(x)$ is a statistical weight for each string $i_{k} i_{k-1}$. $\cdots i_{2} i_{1}$ of measurements.

This equation is consistent with Quantum Mechanics, since $p_{n}\left(M_{2}=0\right)=0$. A semiclassical approximation corresponds to a distribution function peaked around $M_{2} \ll 1$, which brings the fidelity $F_{13}$ close to unity. In fact, the above approximation is an optimization process, rather than the maximization $F_{13}=1$. Consequently, we can see that the semiclassical average fidelity is

$$
\langle F\rangle \sim 2 \pi \sum_{x} \delta_{x, n n n \cdots n n}\left(1-\frac{1}{4} M_{2}^{2}\right)\left(1+M_{2}^{2}\right)
$$

where the Kronecker-delta function is centered around the desired qubit state. Equation (14) follows from substituting in (12) the Taylor expansions of (10) and (13) to next-to-leading order in powers of $M_{2}^{2}$.

\section{Optimization of the Average Fidelity}

Definition 2 The following one-to-one and onto mapping between the set of strings $x \in Z_{2}^{N}$ and the discrete unitary vectors $\vec{x}_{k} \in \mathfrak{R}^{N}$ is defined

$i_{k} i_{k-1} \cdots i_{2} i_{1} \leftrightarrow \vec{x}_{k}=\frac{1}{\left|\vec{x}_{k}\right|}\left(i_{k}, i_{k-1}, \cdots i_{2}, i_{1}\right)$,

where $i_{m}=0,1$ for $m=1,2, \cdots, k-1, k$.

With the above definitions the average fidelity in (14) becomes

$$
\langle F\rangle=\sum_{k=0}^{2^{N}-1} A\left(\vec{x}_{k}\right) \frac{1}{2}\left(1+\sqrt{1-M_{2}\left(\vec{x}_{k}\right)^{2}}\right)\left(1-e^{-M_{2}\left(\vec{x}_{k}\right)^{2}}\right),
$$

where $M_{2}\left(\vec{x}_{k}\right)=\sin \theta_{x_{k}} \cos \varphi_{x_{k}}$.

The value $M_{2}\left(\vec{x}_{k}\right)=0$, which contravenes the nocloning theorem, has a vanishing probability. Values of $M_{2}\left(\vec{x}_{k}\right)$ close to zero have small probabilities. We have thus optimized the Uhlmann-Josza average fidelity. The no-cloning theorem bars maximization of 


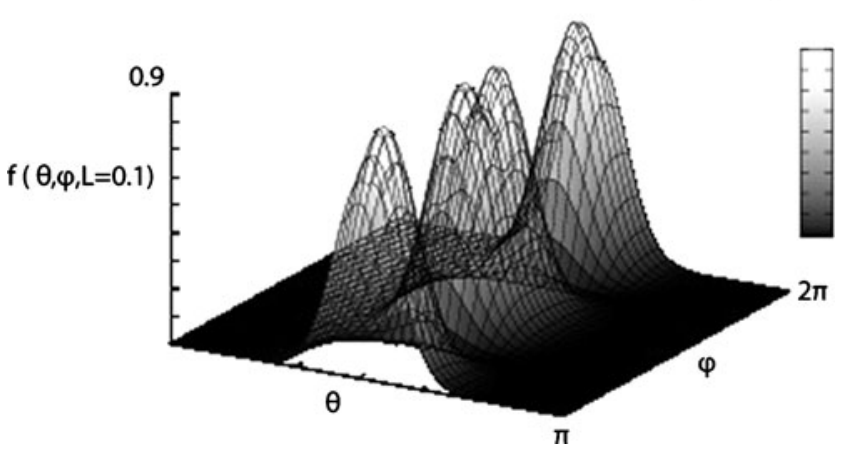

Fig. 1 The function $f(\theta, \varphi, L)$ in (17) for $L=0.1$

this quantity, which would make the probability peak around $M_{2}=0$ (i. e., $F_{13}=1$ ). In order to complete the optimization process we introduce the optimization parameter $L$ through the following

Definition 3 The statistical weight for each outcome $x$ of $N$ measurements is

$A\left(\vec{x}_{k}\right)=e^{\frac{\left(\vec{x}_{k}-\vec{x}_{n}\right)^{2}}{2 L}}$,

where $L$ is the optimization parameter.

From (17) we see that in the limit $2 L \longrightarrow 0$ the function $A\left(\vec{x}_{k}\right)$ becomes a Dirac delta function peaked around the state we want to estimate.

\section{Theoretical Limit: $N \rightarrow \infty$}

Before concluding we consider the case of $N \rightarrow \infty$ measurements of the qubit state. The sum on the right-

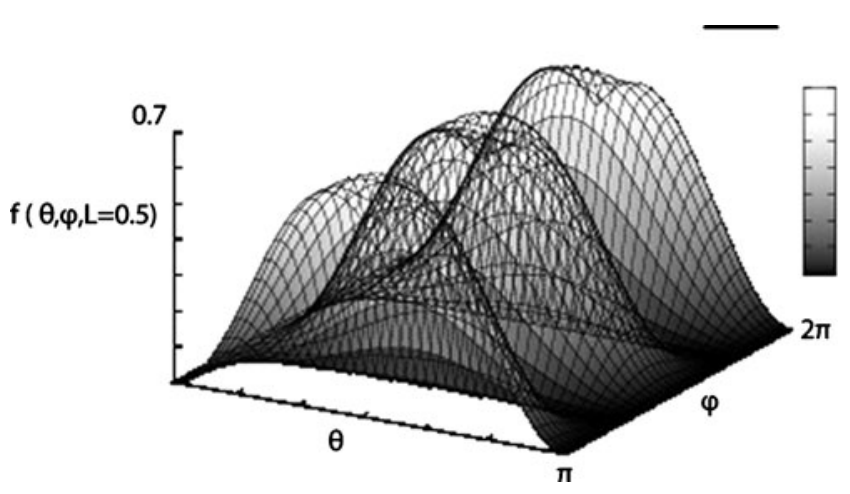

Fig. 2 The function $f(\theta, \varphi, L)$ in (17) for $L=0.5$

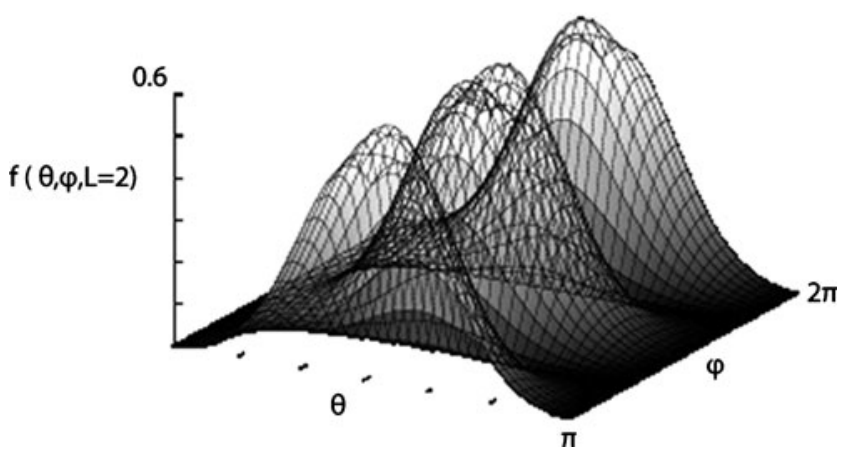

Fig. 3 The function $f(\theta, \varphi, L)$ in (17) for $L=2$

hand side of (16) the becomes the following integral of a distribution function

$$
\begin{aligned}
\lim _{N \rightarrow \infty}\langle F\rangle \rightarrow \int d \Omega f(\theta, \varphi, L) \\
=\frac{1}{2} \int \mathrm{d} \varphi \sin \theta \mathrm{d} \theta e^{\frac{\sin ^{2} \theta}{L}}\left(1+\sqrt{1-\sin ^{2} \theta \cos ^{2} \varphi}\right) \\
\quad \times\left(1-e^{-\sin ^{2} \theta \cos ^{2} \varphi}\right),
\end{aligned}
$$

where we have assumed that $\vec{x} \cdot \vec{x}_{n} \sim \cos \theta$.

Figures 1, 2 and 3 show the theoretical distribution functions, i. e., the distributions for $N \rightarrow \infty$ in the integrand of (18) for $L=0.1,0.5$, and 2, respectively. From Fig. 1 we can see that with a very small optimization parameter $L=0.1$ the distribution function is peaked around the classical value. Figures 2 and 3 show that quantum effects become more important for larger optimization parameters.

\section{Comparison with Collective Schemes}

The distribution function for collective-measurement schemes $[6,21,22]$ is always calculated with the onedegree-of-freedom fidelity $F_{3}(6)$, which is less general than $F_{13}$ - the most general fidelity. The corresponding expression for the average fidelity is, then,

$$
\begin{aligned}
\langle F\rangle_{C M} \longrightarrow & \int_{0}^{1} d x F_{13}(x)+\frac{F_{13}(1)-F_{13}(0)}{2 N} \\
& +\frac{F_{13}^{\prime}(1)-F_{13}^{\prime}(0)}{12 N^{2}}-\cdots
\end{aligned}
$$

This expression is more general than the one used by the common collective schemes, which shed no light on the no-cloning theorem. 


\section{Conclusions}

We have discussed the optimal estimate of a pure qubit state on the basis of the Uhlmann-Josza fidelity. Our analysis respects the limitations imposed by the nocloning theorem. In order to accomplish our objectives we derived a realistic expression for the average fidelity given by (16). In addition, an optimization parameter $L$ was introduced in our formalism. With $L$ close to zero we obtained a fully reconstructed qubit, while larger values represent good quantum optimization of the estimate for the qubit. Thus, $L$ is interpreted as the degree of quantumness of the average fidelity associated with the optimization of the approximate estimation of the qubit-state reconstruction. Quantum Mechanics preventing full reconstruction of a pure qubit state, only estimates of its reconstruction can be obtained. We have therefore used the Uhlmann-Josza fidelity to obtain an optimized estimate of the reconstruction of a qubit.

\section{References}

1. G.P. Berman, G.D. Doolen, V.I. Tsifrinovich, Phys. Rev. Lett. 84, 1615 (2000)

2. A. Biswas, G.S. Agarwal, Phys. Rev. A 69, 062306 (2004)

3. E. Bagan, M. Biag, A. Monras, R. Muñoz-Tapia. Squeezed states and uncertainty relations, in Proceedings of the 8th ICSSUR (Rinton Press, Paramus, 2003)

4. A.S. Holevo, Probabilistic and statistical aspects of quantum theory (North Holland, Amsterdam, 1982)

5. K.R. Jones, Phys. Rev. A 50, 3682 (1994); R.D. Gill, S. Massar, Phys. Rev. A 61, 042312 (2000); D.G. Fisher, S.H. Kienle, M. Freyberger, Phys. Rev. A 61, 032306 (2000); Th. Hannemann, et al., Phys. Rev. A 65, 050303 (2002)

6. E. Bagan, M. Baig, R. Muñoz-Tapia, Phys. Rev. Lett. 89, 277904 (2002)

7. W.K. Wootters, W.H. Zurek, Nature 299, 802 (1982)

8. V. Buzek, M. Hillery, Physics World 14(11), 25 (2001)

9. For clarity we present a brief proof of the no-cloning theorem. Let $|\psi\rangle_{A}$ (representing the system $A$ ) be the stateto be copied and consider an independent state $|i\rangle_{B}$ (representing the system $B$ ). The corresponding composite system is $|\psi\rangle_{A}|i\rangle_{B}$. Let $U=e^{-i H t}$ be the unitary evolution operator, where $H$ is the total Hamiltonian. If $U$ is the copier then it follows that $U|\psi\rangle_{A}|i\rangle_{B}=|\psi\rangle_{A}|\psi\rangle_{B}$ and $U|\phi\rangle_{A}|i\rangle_{B}=|\phi\rangle_{A}|\phi\rangle_{B}$ for all $|\psi\rangle$ and $|\phi\rangle$. Given that the unitary operator $U$ preserves the inner product, one has that $\left\langle\left. i\right|_{B}\left\langle\left.\phi\right|_{A} U^{\dagger} U \mid \psi\right\rangle_{A} \mid i\right\rangle_{B}=\left\langle\left.\phi\right|_{B}\left\langle\left.\phi\right|_{A} \mid \psi\right\rangle_{A} \mid \psi\right\rangle_{B}$, i. e., $\langle\phi \mid \psi\rangle=\langle\phi \mid \psi\rangle^{2}$. This would imply that either $\phi=\psi$ or $\phi$ is orthogonal to $\psi$, which is not the case.

10. One might think that a pure qubit state is a fiction. However, the pure qubit states are often useful for encoding logical qubits in quantum error correction codes theory. For a discussion, see E. Knill, R. Laflamme, Phys. Rev. A 55, 900 (1997)

11. A.G. White, et al., Phys. Rev. Lett. 83, 3102 (1999); G.M. D'Ariano, M.G.A. Paris, Phys. Rev. A 60, 518 (1999); G.M. D'Ariano, P. Lo Presti, Phys. Rev. Lett. 86, 4195 (2001); R.T. Thew, et al., Phys. Rev. A 66, 012303 (2002); J.B. Alepeter, et al., Phys. Rev. Lett. 90, 193601 (2003)

12. Z. Hradil, Phys. Rev. A 55, 1561 (1997); K. Banaszek, Phys. Rev. A 59, 4797 (1999); J. Fiurázek, Z. Hradil, Phys. Rev. A 63, 020101 (2001)

13. This means that $x=\overbrace{i_{N} i_{N-1} i_{N-2} \cdots i_{2} i_{1}}^{N \text { times }}$, where $i_{k}=0,1$. There will then be $2^{N}$ possible results. Here we are implicitly using the Von Neumann projective measurements $[5,6]$.

14. A. Uhlmann, Rep. Math. Phys. 9, 273 (1976)

15. C.A. Fuchs, J. van de Graaf, IEEE Trans. Inf. Theory 45, 1216 (1999)

16. M.A. Nielsen, I. Chuang, Quantum computation and quantum information (Cambridge University Press, Cambridge, 2000)

17. Z.-H. Chen, Z. Ma, F.-L. Zhang, Cent. Eur. J. Phys. 9, 1036 (2009)

18. I. Bengtsson, K. Zyczkowski, Geometry of quantum states: an introduction to quantum entanglement (Cambridge University Press, Cambridge, 2008)

19. J. Audretsch, Entangled systems: new directions in quantum physics (Wiley-VCH, Berlin, 2007)

20. S. Massar, D. Popescu, Phys. Rev. Lett. 74, 1259 (1995)

21. R. Derka, V. Buzek, A. Ekert, Phys. Rev. Lett. 80, 1571 (1998)

22. E. Bagan, M. Baig, R. Muñoz-Tapia, Phys. Rev. Lett. 89, 277904 (2002)

23. J.I. Latorre, P. Pascual, R. Tarrach, Phys. Rev. Lett. 81, 1351 (1998); N. Gisin, S. Popescu, Phys. Rev. Lett. 83, 432 (1999); E. Bagan, et al., Phys. Rev. Lett. 85, 5230 (2000); A. Acin, J.I. Latorre, P. Pascual, Phys. Rev. A 61, 022305 (2000); A. Peres, P.F. Scudo, Phys. Rev. Lett. 86, 4160 (2001) 